\title{
Erste Langzeitstudie: Botulinumtoxin bessert Läuferknie
}

Zu den arzneimittelrechtlich zugelassenen Indikationen von Botulinumneurotoxin A (BoNT-A) gehört die Behandlung der Spastik der Beinmuskulatur infolge eines Schlaganfalls oder einer infantilen Zerebralparese. Die intramuskuläre Injektion von BoNT-A wird gelegentlich auch als Off-Label-Therapie zur gezielten Schwächung hypertrophierter Muskeln bei Engpasssyndromen (zum Beispiel Musculus scalenus anterior bei neurogenem Thoracic-Outlet-Syndrom, Musculus piriformis bei Piriformis-Syndrom) eingesetzt. In der Sportmedizin spielt BoNT-A bislang eine untergeordnete Rolle.

E inige seit 2006 publizierte Pilotstudi¿ en, davon eine kleine randomisierte Studie, sprechen für einen günstigen Effekt der einmaligen EMG-gesteuerten BoNT-A-Injektion in den Musculus vastus lateralis beim chronischen, therapierefraktären patellofemoralen Schmerzsyndrom (PFSS).

Das PFSS (Synonym: Chondropathia patellae, parapatellares Schmerzsyndrom, Chondromalacia patellae) ist ein uneinheitliches Krankheitsbild, das durch eine Über- oder Fehlbelastung der proximalen Beinmuskulatur ausgelöst wird. Neben einer entzündlichen Reizung des Tractus iliotibialis (iliotibiales Bandsyndrom [ITBS], Synonym: Läuferknie, Tractus-Syndrom) kann ursächlich auch eine Bursitis oder Tendinitis dem PFSS zugrunde liegen. Daher waren Patienten mit Bursitis, Tendinitis, hypermobiler Patella, früherer Patelladislokation oder deutlichem Übergewicht (BMI > 30) von diesen Pilotstudien ausgeschlossen worden, sodass nur Patienten mit einem chronischen ITBS mit BoNT-A behandelt wurden.

Bislang ist unklar, ob die BoNT-A-Injektion in den Musculus vastus lateralis oder tensor fasciae latae erfolgreicher ist. Zudem liegen kaum Daten über die Langzeitwirkung dieser Behandlung vor. Die Studie von Stephen und Kollegen untersucht erstmals über einen Zeitraum von fünf Jahren in einer einarmigen prospektiven Studie die Effektivität der ultraschallgezielten BoNT-A-Injektion in den Musculus tensor fasciae latae bei Patienten mit ITBS. Eingeschlossen wurden 45 erwachsene Patienten mit ei- nem aktivitätsassoziierten ITBS mit einer Symptomdauer von mindestens drei Monaten. Die Diagnose musste mittels MRT des Oberschenkels belegt worden sein. Der Knieschmerz musste durch Anspannung des iliotibialen Trakts im modifizierten Ober-Test reproduzierbar auslösbar und eine mindestens sechswöchige Physiotherapie musste erfolglos geblieben sein. Ausschlusskriterien waren eine Subluxation, Dislokation oder Tendinopathie der Patella sowie eine ausgeprägte patellofemorale Osteoarthritis.

Alle Patienten erhielten ultraschallgesteuert eine Injektion von 75 I.E. BoNT$\mathrm{A}$ in $0,75 \mathrm{ml}$ isotonischer Kochsalzlösung in den zentralen Bauch des Musculus tensor fasciae latae. Die anschließenden standardisierten Untersuchungen und Behandlungen führten spezialisierte Physiotherapeuten durch. Alle Patienten wurden vor der Behandlung sowie nach einer, vier und zwölf Wochen und nochmals nach fünf Jahren untersucht. Der primäre Endpunkt war die Selbstbeurteilung des Knieschmerzes anhand der Anterior Knee Pain Scale (AKPS; Kujala-Score). Dabei handelt es sich um einen Fragebogen mit 13 kniebezogenen Fragen und einem maximalen Score von 100, wobei höhere Scores geringere Beschwerden bedeuten. Als sekundärer Endpunkt war die Länge des Tractus iliotibialis definiert worden, bestimmt mit dem modifizierten Ober-Test.

Die Fünf-Jahres-Kontrolle konnte bei 39 der 45 Patienten durchgeführt werden. Als wesentliches Ergebnis zeigte sich eine über fünf Jahre anhaltende
Besserung des Knieschmerzes in der AKPS, mit einem mittleren initialen Score von 61 und einem Score von 87 nach fünf Jahren. Zudem zeigte sich eine signifikante Verlängerung des iliotibialen Trakts.

Mittels Computersimulation wurde eine Aktivitätsnormalisierung des Musculus gluteus medius infolge der dosierten Schwächung des Musculus tensor fasciae latae ermittelt. Vier Patienten erhielten ein oder zwei weitere BoNT-AInjektionen, die dann zu einem Sistieren der Kniebeschwerden führten. Lediglich bei zwei Patienten waren nur Kurzzeiteffekte zu erreichen, sodass sie schließlich operiert werden mussten. $69 \%$ der Patienten wurden völlig schmerzfrei. Ebenfalls 69 \% konnten sportliche Aktivitäten wieder auf dem früheren Niveau fortführen.

Fazit: Erstmals konnte in einer prospektiven Langzeitstudie gezeigt werden, dass die ultraschallgesteuerte BoNT-AInjektion in den Muskulus tensor fasciae latae einen anhaltenden Behandlungserfolg bei erwachsenen Patienten mit therapieresistentem ITBS erbringt. Allerdings ist die Replikation dieser Ergebnisse in weiteren, möglichst randomisierten Studien erforderlich, bevor eine breite Anwendung von BoNT-A in dieser Indikation empfohlen werden kann.

\section{Prof. Dr. med. Uwe Walter}

Stephen JM et al. The use of sonographically guided botulinum toxin type A (Dysport) injections into the tensor fasciae latae for the treatment of lateral patellofemoral overload syndrome. Am J Sports Med. 2016; 44 (5): 1195-202 